\title{
microRNA-144 inhibits cell proliferation and invasion by directly targeting TIGAR in esophageal carcinoma
}

\author{
YUSHU MU ${ }^{1 *}$, QIFEI WANG ${ }^{1 *}$, LEI TAN $^{2}$, LIN LIN $^{3}$ and BENHUAZHANG ${ }^{4}$ \\ ${ }^{1}$ Department of Thoracic Surgery, Affiliated Hospital of Taishan Medical University; Departments of ${ }^{2}$ Thoracic Surgery \\ and ${ }^{3}$ Digestive Medicine, Tai'an City Central Hospital; ${ }^{4}$ Department of Oncology, Affiliated Hospital \\ of Taishan Medical University, Tai'an, Shandong 271000, P.R. China
}

Received September 1, 2018; Accepted October 18, 2019

DOI: $10.3892 / \mathrm{ol} .2020 .11420$

\begin{abstract}
RNAs (miRNAs) have been identified to play vital roles in the development and progression of numerous different types of human malignancy, including esophageal squamous cell carcinoma (ESCC). In the present study, the biological function of microRNA-144 (miR-144) was investigated, as well as its underlying molecular mechanism in ESCC. The results revealed that miR-144 expression was significantly decreased, whereas the expression of TP53-inducible glycolysis and apoptosis regulator (TIGAR) was significantly increased in human ESCC tissues when compared with adjacent non-tumor tissues. An increase in TIGAR was significantly associated with tumor size and Tumor-Node-Metastasis staging in patients. Functional analysis revealed that the overexpression of miR-144 using lentivirus particles significantly inhibited cell proliferation and tumor colony formation, and induced cell apoptosis in EC9706 and EC109 cells. The autophagy activity was also enhanced by miR-144 activity. In addition, overexpression of miR-144 significantly inhibited tumor growth in vivo. In the present study, TIGAR was confirmed to be the downstream target of miR-144 in ESCC. siRNA-mediated downregulation of TIGAR inversely regulated the inhibition effect of miR-144 on ESCC cells. To conclude, the present study demonstrated that miR-144 inhibits proliferation and invasion in esophageal cancer by directly targeting TIGAR.
\end{abstract}

\section{Introduction}

Esophageal cancer (EC) is one of the most malignant types of tumor in humans (1-4). As the eighth most common type of cancer worldwide, the yearly estimated number of new

Correspondence to: Dr Benhua Zhang, Department of Oncology, Affiliated Hospital of Taishan Medical University, 706 Taishan Street, Tai'an, Shandong 271000, P.R. China

E-mail: benhuatmu@163.com

${ }^{*}$ Contributed equally

Key words: microRNA-144, esophageal carcinoma, TP53-inducible glycolysis and apoptosis regulator
EC cases in the USA alone is $>16,000$, with an estimated $>150,000$ cases of EC-associated mortality each year (1-4). Histologically, as the two major subtypes of EC, esophageal adenocarcinoma and esophageal squamous carcinoma (ESCC) represent $\sim 90 \%$ of all EC cases (1,5-7). Among them, ESCC is the most dominant subtype in developing countries, including China $(8,9)$. Despite advances in the early diagnosis, comprehensive therapy and surgical techniques, the prognosis for patients with ESCC remains far from satisfactory, with an overall 5-year survival rate of $<25 \%$ (10-12). Therefore, it is essential that the molecular mechanisms underlying the neoplastic progression of ESCC be elucidated, and that novel molecular markers associated with ESCC are identified, in order to facilitate the development of an effective diagnostic and therapeutic strategy for ESCC.

Due to the vital regulatory effects in cell proliferation, apoptosis and differentiation, microRNAs (miRNAs) have been widely studied and proven to be associated with carcinogenesis and tumor progression, and even the response to therapy $(2,3,13,14)$. As a large class of conserved 20-22 nucleotides long small non-coding single stranded RNAs, miRNAs negatively regulate gene expression through targeting the 3'untranslated region (3'UTR) of mRNAs. The aberrant expression of several miRNAs has been identified in $\operatorname{ESCC}(5,15,16)$. Chen et al (6) reported that miR-26b regulates the proliferation, migration and cell cycle transition of cancer cells by suppressing tumor necrosis factor receptor associated factor 5 in ESCC. Fan et al (17) revealed that miR-125b inhibits cell proliferation and induces cell apoptosis in ESCC by targeting Bcl 2 modifying factor. In another clinical study by Dong et al (16), miR-216 a/b was demonstrated to be inversely correlated with lymph node metastasis and the Tumor-Node-Metastasis (TNM) stage (18) of ESCC, which indicated that plasma miRNA-216a/b may serve as potential biomarkers for the diagnosis of ESCC, and that dysregulation of $\mathrm{miR}-216 \mathrm{a} / \mathrm{b}$ may be involved in the progression of ESCC. Collectively, these data indicate that miRNAs are important regulators of ESCC and have been recognized as biomarkers for the diagnosis of ESCC.

miR-144 has been demonstrated to act as a tumor suppressor in multiple types of human malignancy, such as non-small cell lung, gastric, papillary thyroid, bladder and colorectal cancers (19-22). In chronic and acute myeloid leukemia, the expression of miR-144 was also demonstrated 
to be downregulated $(19,20)$. Furthermore, using principal component regression analysis, Gao et al (21) reported that the miR-144/451 cluster may play an important role in the progression of ESCC and may also be considered as a biomarker for the detection of ESCC at an early stage of disease. In another study, Wu et al (22) demonstrated that miRNA-144 was highly expressed in the saliva of patients with EC and can be used as a genetic marker for the early diagnosis of these patients. However, the detailed role of miR-144 and the molecular mechanism underlying ESCC remains unknown and requires elucidation. In the present study, the role of miR-144 in ESCC and the underlying molecular mechanism both in vitro and in vivo are reported.

\section{Materials and methods}

Patients and sample collection. The present study was approved by the Medical Ethics Committee of the Affiliated Hospital of Taishan Medical University (Tai'an, China). Written informed consent was obtained from all patients included within the present study. In total, 80 patients with ESCC were recruited in the present study between January 2017 and November 2018. The inclusion criteria of the present study were as follows: Patients who underwent ESCC surgical resection without prior chemotherapy or radiotherapy. The exclusion criteria were as follows: Patients without confirmed diagnosis of ESCC or patients with ESCC who had previously received chemotherapy, radiotherapy or biological medication (monoclonal antibodies). ESCC tissues and corresponding normal tissues ( $<5 \mathrm{~cm}$ away from the ESCC tissues) were collected during surgery and immediately stored in liquid nitrogen. Patient diagnoses was validated blindly by pathologists from the Department of Pathology, Affiliated Hospital of Taishan Medical University (Tai'an, China).

Cell lines and cell culture. The ESCC cell line EC109 and EC9706 were purchased from Changsha Yingrun Biotechnology Co., Ltd. The cells were grown in DMEM (Gibco; Thermo Fisher Scientific, Inc.) supplemented with $10 \%$ fetal bovine serum (Gibco; Thermo Fisher Scientific, Inc.), $1 \%$ streptomycin and $1 \%$ penicillin. Cells were cultured in a humidified atmosphere of $5 \% \mathrm{CO}_{2}$ at $37^{\circ} \mathrm{C}$ and the medium was replaced every 3 days.

RNA extraction and reverse transcription-quantitative (RT-q) $P C R$. Total RNA was extracted from tissue samples and ESCC cell lines using a TRIzol ${ }^{\circledR}$ kit (Invitrogen; Thermo Fisher Scientific, Inc.) and a RNeasy Mini kit (Qiagen, Inc.). RNA was reverse transcribed into cDNA using an miScriptRT kit (Qiagen $\mathrm{GmbH}$ ). The protocol for reverse-transcription was as follows: $37^{\circ} \mathrm{C}$ for $60 \mathrm{~min}$ followed by $85^{\circ} \mathrm{C}$ for $5 \mathrm{sec}$. qPCR was subsequently performed using a TaqMan miRNA PCR kit (Applied Biosystems; Thermo Fisher Scientific, Inc.) in an ABI 7500 Real-Time PCR system (Invitrogen; Thermo Fisher Scientific, Inc.), according to the manufacturer's protocol. The following primer sequences were used for the qPCR: miR-144; Forward, 5'-TACAGTATAGATGAT-3' and reverse, 5'-GTGCAGGGTCCGAGGT-3'; TIGAR; Forward, 5'-ACT CAAGACTTCGGGAAAGGA-3', and reverse, 5'-CACGCA TTTTCACCTGGTCC-3'; U6; Forward, 5'-CTCGCTTCG
GCAGCACA-3' and reverse, 5'-AACGCTTCACGAATT TGCGT-3'; $\beta$-actin; Forward, 5'-CTCCATCCTGGCCTC GCTGT-3', and reverse, 5'-GCTGTCACC TTCACCGTT CC-3'. The following thermocycling conditions were used for the PCR: initial denaturation at $95^{\circ} \mathrm{C}$ for $30 \mathrm{sec} ; 40$ cycles PCR extension of $95^{\circ} \mathrm{C}$ for $3 \mathrm{sec}$ and $60^{\circ} \mathrm{C}$ for $30 \mathrm{sec}$. Small nuclear RNA U6 (U6) (Fig. 1A) and $\beta$-actin (Fig. 1B) were used as the internal reference genes for miR-144 and TIGAR PCR analysis, respectively. mRNA levels were calculated as fold-changes using the $2^{-\Delta \Delta \mathrm{Cq}}$ method (23).

Cell transfection. The overexpression lentiviral particles $\left(10^{8} \mathrm{TU} / \mathrm{ml}\right)$ of miR-144-3p were purchased from GenePharma, Inc. 293FT cells (American Type Culture Collection) were used as the interim cell line to generate the packaged lentivirus. The sequences of miR-144-3p were as follows: Forward, 5'-GCGCGAATTCGAGATCTTAACAGACCCTAGCTC-3' and reverse, 5'-GCGCGGATCCGTGCCCTGGCAGTCAGT AGG-3'. In detail, lentivirus particles were generated using co-transfection of pCDH-miR-144-3p or pCDH with psPAX2 and pMD2G vectors into 293FT cells. Cells were transfected with Lipofectamine ${ }^{\circledR} 3000$ reagent (Invitrogen; Thermo fisher Scientific, Inc.). EC109 and EC9706 cells were transfected into three different groups: Blank group, miR-144 negative control (miR-NC with empty lentivirus packing vector) and miR-144 overexpression group, according to the manufacturer's protocol that supplied the miR-144-3p lentiviral particles (GenePharm, Inc.). Following transfection, the expression of miR-144 was determined via RT-qPCR, as aforementioned. Cells were collected for further experiments $48 \mathrm{~h}$-post transfection. The targeting siRNA-TIGAR and control siRNA $(20 \mu \mathrm{M}$, respectively) were also purchased from GenePharma, Inc., and the sequences were as follows: siRNA-TIGAR forward, 5'-CCUACACCAUCAUCUAAAUTT-3' and reverse, 5'-AUU UACAUCAUCCUGUACCTT-3' and control siRNA forward, 5'-UUCUCCCAACGUCUCACCUTT-3', and reverse, 5'-ACG UCACACGUUCGCAGAATT-3'.

Cell proliferation assay. The effect of miR-144 on the proliferation of ESCC cells was evaluated using a Cell Counting Kit-8 (CCK-8; Beyotime Institute of Biotechnology). In detail, EC109 and EC9706 cells (2x10 4 cells/well in $100 \mu 1$ medium) were seeded into 96 -well plates. After $24 \mathrm{~h}$ of incubation at $37^{\circ} \mathrm{C}$, the cells were transfected with miR-144 overexpression lentiviral particles, negative control lentiviral particles or blank medium for $48 \mathrm{~h}$ and incubated in a humidified atmosphere of $5 \% \mathrm{CO}_{2}$ at $37^{\circ} \mathrm{C}$ for $0,24,48,72$ and $96 \mathrm{~h}$ following transfection. Subsequently, $15 \mu \mathrm{l}$ of CCK-8 reagent was added to each well. After $4 \mathrm{~h}$ incubation at $37^{\circ} \mathrm{C}$, the absorbance values at $450 \mathrm{~nm}$ were measured using a microplate reader (Bio-Rad Laboratories, Inc.). Each experiment was performed in triplicate.

Tumor colony formation assay. EC109 and EC9706 cells were transfected with miR-144 overexpression lentiviral particles, negative control lentiviral particles or blank medium, and trypsinized $24 \mathrm{~h}$ post-transfection. Each well had $2 \times 10^{3}$ EC109 and EC9706 cells added to the 6-well plates and were incubated at $37^{\circ} \mathrm{C}$ for 7 days. The colonies were fixed with $4 \%$ methanol for $15 \mathrm{~min}$ at room temperature and stained with crystal violet for 
A

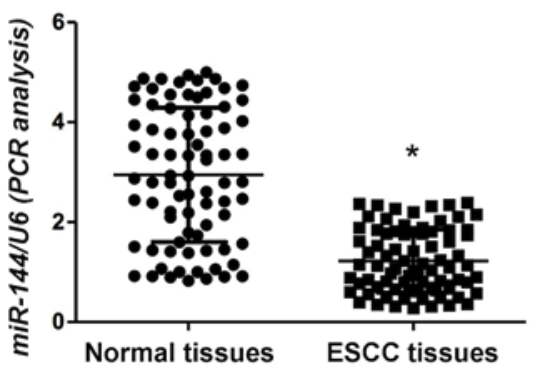

B

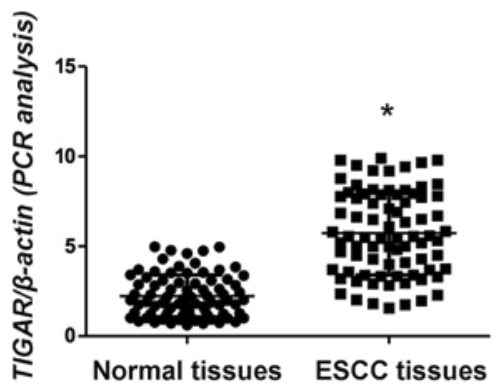

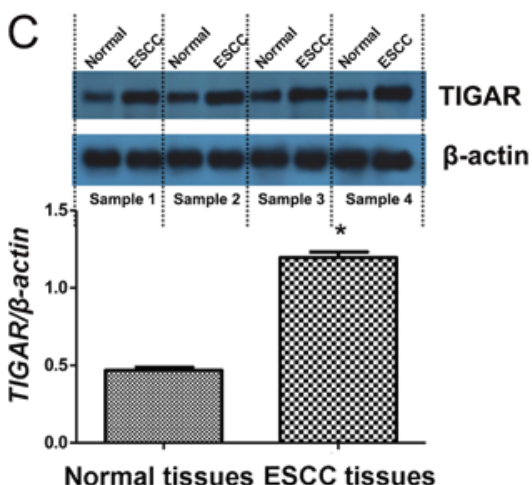

Figure 1. miR-144 is downregulated while TIGAR is upregulated in human ESCC. (A) PCR analysis revealed that the expression of miR-144 was significantly downregulated in ESCC tissues compared with the adjacent normal tissues. (B) PCR analysis of 80 samples revealed significantly higher mRNA expression levels of TIGAR in ESCC tissues compared with the adjacent normal tissues. (C) Western blot analysis of four randomly selected ESCC samples together with the adjacent noncancerous tissues confirmed that the protein expression of TIGAR in ESCC tissues was higher than the adjacent normal tissues. ${ }^{*} \mathrm{P}<0.05$ vs. control. miR, microRNA; TIGAR, TP53-inducible glycolysis and apoptosis regulator; ESCC, esophageal squamous cell carcinoma.

10 min at $37^{\circ} \mathrm{C}$. Images were captured using a digital camera in order to assess the number of stained tumor cell colonies containing at least 50 cells. ImageJ software (version 1.47 ; National Institutes of Health) was used to quantify the results of tumor colony formation assay.

Bioinformatics. miRNA target gene predictions were taken from TargetScan (version 7.0) (24), which predicts the biological targets of miRNAs by searching for the presence of 8,7 and 6 mer sites that match the seed region of each miRNA (25). The prediction of miRNA functions was determined using DIANA-miRPath (version 3.0; microrna.gr/miRPathv3) (26).

Cell apoptosis assay. In order to quantify the cell apoptosis rates, a flow Annexin V-FITC Apoptosis Detection kit (BD Biosciences) was applied according to the manufacturer's protocols. In brief, the transfected ESCC cells were collected and resuspended in $500 \mu \mathrm{l}$ Annexin V-binding buffer (BD Biosciences). Subsequently, $5 \mu \mathrm{l}$ of FITC-conjugated Annexin $\mathrm{V}$ and $5 \mu \mathrm{l}$ propidium iodide were added. Stained cell fluorescence was analyzed using the FACSCalibur flow cytometer (Beckman Coulter, Inc.) following incubation for $10 \mathrm{~min}$ at room temperature in the dark. The results of flow Annexin V-FITC Apoptosis Detection kit were analyzed using FlowJo software (version 9.6.2; FlowJo LLC). The caspase-3/-7 activity apoptosis assay was performed using a caspase-3/-7 Activity Apoptosis Assay kit (https://www.aatbio.com). ESCC cells were incubated with caspase-3/-7 assay solution (AAT Bioquest) for $1 \mathrm{~h}$ at room temperature. Fluorescence intensity was detected at $\mathrm{Ex} / \mathrm{Em}=490 / 525 \mathrm{~nm}$ using a fluorescence microplate reader (Tecan Group, Ltd.).

Western blot analysis. The western blot analysis was performed as previously described $(27,28)$. In brief, total protein was extracted using protein lysis buffer (Beyotime Institute of Biotechnology) and quantified using a bicinchoninic acid assay assay kit (Bio-Rad Laboratories, Inc.). A total of $30 \mu \mathrm{g}$ protein/lane was separated via SDS-PAGE on a $10 \%$ gel. The separated proteins were subsequently transferred onto a polyvinylidene difluoride membrane (EMD Millipore) and blocked in $5 \%$ non-fat dried milk for $2 \mathrm{~h}$ at room temperature. The membrane were incubated with primary antibodies against TIGAR (1:1,000; cat. no. 14751) and $\beta$-actin $(1: 8,000$; cat. no. 4970) (both from Cell Signaling Technology, Inc.) overnight at $4^{\circ} \mathrm{C}$. Following the primary incubation, membranes were incubated with a horseradish peroxidase-conjugated donkey anti-goat IgG secondary antibody (1:1,000; cat. no. A0181; Beyotime Institute of Biotechnology) for $2 \mathrm{~h}$ at room temperature. Protein bands were visualized using enhanced chemiluminescence reagents (Pierce; Thermo Fisher Scientific, Inc.) and analyzed using Bio-Rad Quantity One software (version 4.4.0; Bio-Rad Technologies, Inc.).

Construction of 3'UTR reporter plasmid and luciferase assay. The 3'UTR of TIGAR, which contains a putative target region for miR-144, was amplified via PCR. The primer sequences were as follows: Forward, 5'-GTAGAGCTCTTAACTTAT TTTGTCCAAGTACAGTT-3', and reverse, 5'-GAACTC GAGAAATTCCAATAGTTGATTTATACGTA-3'. The thermocycling conditions were as follows: Initial denaturation at $94^{\circ} \mathrm{C}$ for $3 \mathrm{~min} ; 35$ cycles of $94^{\circ} \mathrm{C}$ for $30 \mathrm{sec}, 55^{\circ} \mathrm{C}$ for $30 \mathrm{sec}$ and $72^{\circ} \mathrm{C}$ for $35 \mathrm{sec}$. Takara $\mathrm{Taq}^{\mathrm{TM}}$ DNA polymerase (Takara Bio, Inc.) was used for the PCR. The TIGAR 3'UTR mutant construct was generated via overlap extension PCR $\left(72^{\circ} \mathrm{C}\right.$ for $\left.3 \mathrm{~min}\right)$. Fragments were inserted between the XbaI and XhoI sites in the pmirGLO (Promega Corporation). Co-transfection of the reporter vector (pmirGLO-wt-TIGAR or pmirGLO-mut-TIGAR) and miRNA (miR-144 mimics or scramble) was performed using Lipofectamine ${ }^{\mathrm{TM}} 2000$ (Invitrogen; Thermo Fisher Scientific, Inc.). After $24 \mathrm{~h}$ transfection, firefly and Renilla luciferase activities were measured using the Dual-Luciferase Reporter Assay kit (Promega Corporation), according to the manufacturer's protocol.

Tumor growth assay. All in vivo animal studies were conducted using a protocol approved by the Animal Care and Use Committee of the Affiliated Hospital of Taishan Medical University (Tai'an, China). A total of 15 male nude mice (5-weeks-old) weighing $25 \pm 5 \mathrm{~g}$ at the beginning of the study were purchased from the Laboratory Animal Centre of 
Table I. Clinical profiles of the 80 patients with esophageal squamous cell carcinoma.

\begin{tabular}{|c|c|c|c|c|c|}
\hline Clinicopathological feature & Number of cases & $\operatorname{miR}-144$ & P-value & TIGAR & P-value \\
\hline \multicolumn{6}{|l|}{ Sex } \\
\hline Male & 44 & 1.23 & \multirow[t]{2}{*}{0.847} & 5.68 & \multirow[t]{2}{*}{0.764} \\
\hline Female & 36 & 1.21 & & 5.79 & \\
\hline \multicolumn{6}{|l|}{ Age } \\
\hline$\leq 60$ years & 43 & 1.18 & \multirow[t]{2}{*}{0.496} & 5.87 & \multirow[t]{2}{*}{0.509} \\
\hline$>60$ years & 37 & 1.27 & & 5.56 & \\
\hline \multicolumn{6}{|l|}{ Tumor size } \\
\hline$\leq 3 \mathrm{~cm}$ & 56 & 1.22 & \multirow[t]{2}{*}{0.745} & 4.59 & \multirow[t]{2}{*}{$<0.001$} \\
\hline$>3 \mathrm{~cm}$ & 24 & 1.23 & & 8.38 & \\
\hline \multicolumn{6}{|l|}{ Grading } \\
\hline $\mathrm{H}$ & 23 & 1.13 & \multirow[t]{3}{*}{0.471} & 5.07 & \multirow[t]{3}{*}{0.181} \\
\hline M & 27 & 1.33 & & 6.29 & \\
\hline $\mathrm{L}$ & 30 & 1.20 & & 5.73 & \\
\hline \multicolumn{6}{|l|}{ Metastasis } \\
\hline Present & 44 & 0.72 & \multirow[t]{2}{*}{$<0.001$} & 5.84 & \multirow[t]{2}{*}{0.575} \\
\hline Absent & 36 & 1.83 & & 5.59 & \\
\hline \multicolumn{6}{|l|}{ TNM staging } \\
\hline I & 21 & 1.90 & \multirow[t]{4}{*}{$<0.001$} & 5.64 & \multirow[t]{4}{*}{0.010} \\
\hline II & 26 & 1.36 & & 6.12 & \\
\hline III & 21 & 0.73 & & 6.25 & \\
\hline IV & 12 & 0.58 & & 4.10 & \\
\hline
\end{tabular}

Mean values of miR-144 and TIGAR are presented in this table. miR, microRNA; TIGAR, TP53-inducible glycolysis and apoptosis regulator; $\mathrm{H}$, high; M, moderate; L, low; TNM, Tumor-Node-Metastasis.

Affiliated Hospital of Taishan Medical University and divided into three groups $(\mathrm{n}=5$ in each group) including blank group, miR-NC group and miR-144 group. All mice were kept at $25^{\circ} \mathrm{C}$ clean atmosphere with $50 \%$ relative humidity and a $12 / 12 \mathrm{~h}$ dark/light cycle. The mice had unlimited access to food and water. EC9706 with blank treated or stably transfected with miR-NC or miR-144 lentivirus $\left(1.5 \times 10^{6}\right.$ in $\left.0.2 \mathrm{ml}\right)$ were injected subcutaneously into the right dorsal flank of each mouse. Tumor size was calculated using the following formula: volume $=\left[0.5 \mathrm{x}\left(\right.\right.$ length $\mathrm{x}$ width $\left.\left.{ }^{2}\right)\right]$ at 1-, 2-, 3- and 4-weeks after inoculation of tumor cells. Mice were sacrificed using cervical dislocation at 4-weeks after inoculation of tumor cells.

Immunohistochemistry (IHC). The mouse tumor tissues were fixed in $4 \%$ paraformaldehyde for $48 \mathrm{~h}$ at room temperature and embedded in paraffin. Paraffin-embedded tissue samples were cut into $4-\mu \mathrm{m}$-thick sections. IHC analysis was subsequently carried out using standard techniques. In brief, deparaffinized sections were incubated with $3 \% \mathrm{H}_{2} \mathrm{O}_{2}$ for 10 min at room temperature, followed by $0.1 \%$ trypsin (Beyotime Institute of Biotechnology) for $20 \mathrm{~min}$ at room temperature. Subsequently, sections were incubated with the primary antibody against Ki-67 (1:800; cat. no. 9449; Cell Signaling Technology, Inc.) overnight at $4^{\circ} \mathrm{C}$, followed by incubation with a HRP-labeled goat anti-mouse IgG secondary antibody (1:50; cat. no. A0216; Beyotime Institute of Biotechnology) for $2 \mathrm{~h}$ at $37^{\circ} \mathrm{C}$. IHC staining was visualized using a DAB horseradish peroxidase color development kit (Beyotime Institute of Biotechnology) and images were captured using an Olympus Provis AX70 light microscope (magnification, x400). At least three sections from each specimen were analyzed blindly by two independent observers from Department of Pathology, Affiliated Hospital of Taishan Medical University (Tai'an, China). The percentage of Ki-67 positive cells was calculated after counting two hundred cells per region.

Statistical analysis. All experiments were repeated at least three times independently. Data are presented as the mean \pm standard deviation. The results were statistically analyzed using an unpaired two-tailed Student's t-test or oneway analysis of variance (ANOVA) followed by Bonferroni's post hoc test using the SPSS software (version 23.0; IBM Corp.). For the data presented in Table I, an unpaired two-tailed Student's t-test was used to compare the results among two groups, while ANOVA followed by Bonferroni's post hoc test was applied for the comparison among $>2$ groups. $\mathrm{P}<0.05$ was considered to indicate a statistically significant result.

\section{Results}

miR-144 is downregulated and TIGAR is upregulated in human ESCC specimens. The expression levels of miR-144 in clinical ESCC tissues and adjacent tissues were determined via RT-qPCR while the expression of TIGAR was evaluated 

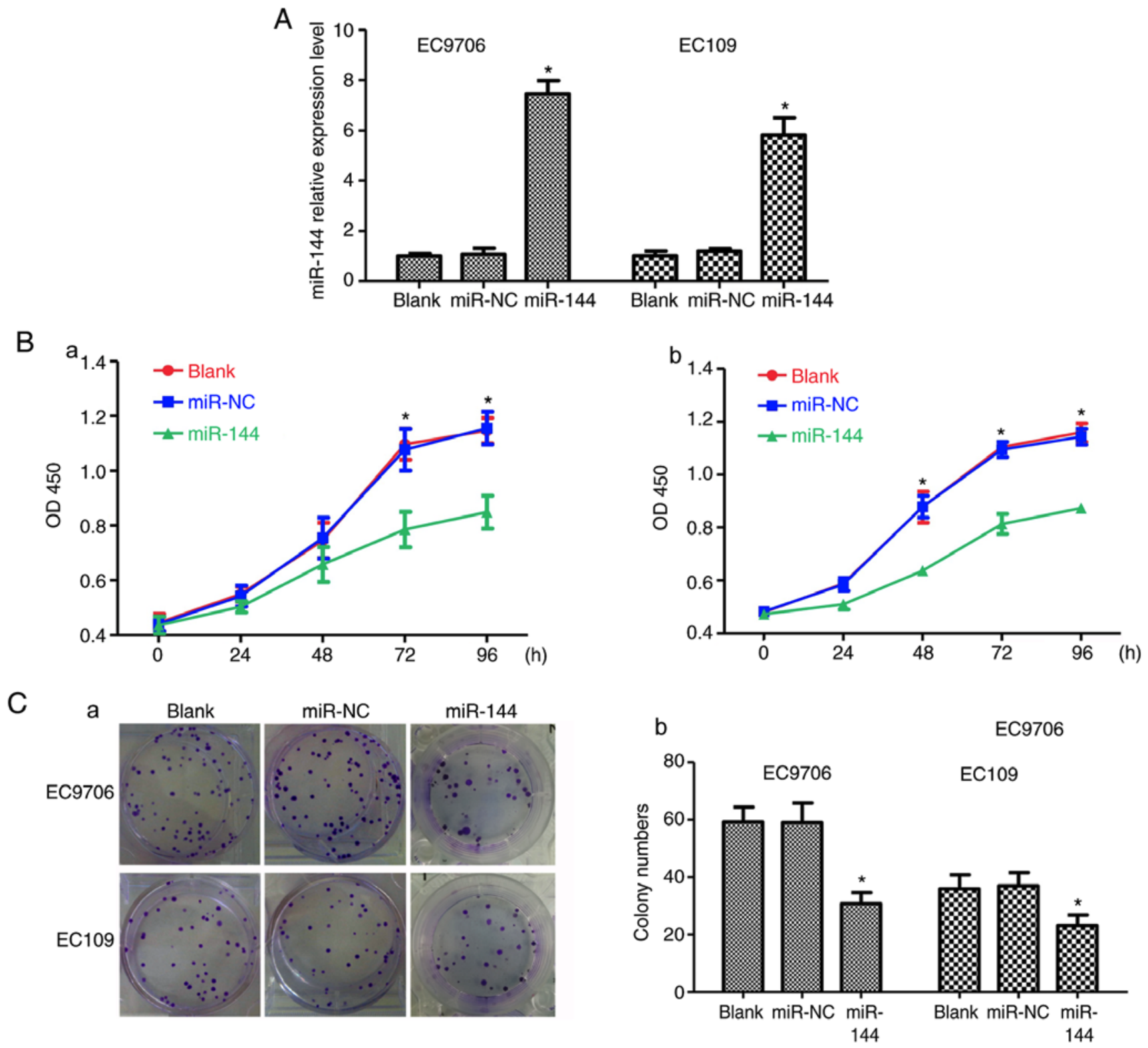

Figure 2. miR-144 suppresses cell proliferation and tumor formation and promotes cell apoptosis in EC9706 and EC109 cells. (A) miR-144 expression levels were upregulated in ESCC cells following lentiviral transfection. (B) (a-b) Overexpression of miR-144 significantly decreased cell proliferation in EC109 and EC9706 cells compared with the miR-NC group and the blank group, respectively. (C) (a-b) Overexpression of miR-144 significantly inhibited the tumor formation ability of EC109 and EC9706 cells. "P<0.05 vs. control. miR, microRNA; ESCC, esophageal squamous cell carcinoma; NC, negative control.

via RT-qPCR and western blot analysis using TIGAR-specific antibodies. Compared with the adjacent non-cancer tissues, the expression of miR-144 was significantly downregulated in ESCC tissues (Fig. 1A). In addition, there were significantly higher mRNA and protein expression levels of TIGAR observed in ESCC tissues compared with the adjacent normal tissues $(\mathrm{P}<0.05$; Fig. $1 \mathrm{~B}$ and $\mathrm{C})$.

Expression levels of miR-144 and TIGAR are associated with ESCC progress. The baseline characteristic of all patients included in the present study are presented in Table I. There was no statistically significant association observed regarding the miR-144, TIGAR expression and sex, age and tumor grading. However, miR-144 expression was significantly associated with ESCC metastasis and TNM staging. In addition, TIGAR expression was significantly associated with ESCC tumor size and TNM staging. These results indicated that the expression levels of miR-144 and TIGAR were closely associated with ESCC progress (Table I).

miR-144 suppresses the proliferation and tumor formation of ESCC cells. In order to investigate the effect of miR-144 on ESCC, human EC9706 and EC109 cells were transfected with miR-144 lentivirus, negative control lentivirus and the blank control. The successful transfection was confirmed by RT-qPCR (Fig. 2A). As presented in Fig. 2B-a and b, overexpressed miR-144 significantly decreased cell proliferation in EC109 and EC9706 cells, compared with the miR-NC group and the blank group. Furthermore, by conducting a tumor colony formation assay, it was revealed that miR-144 significantly inhibited the tumor formation ability of EC109 and EC9706 cells (Fig. 2C-a and b). 
a

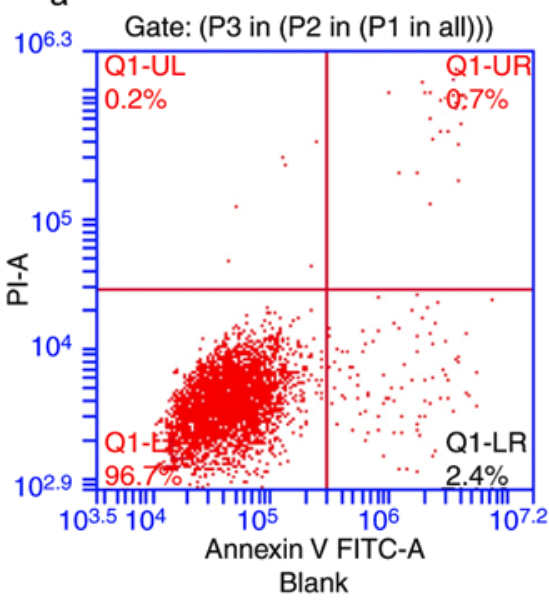

b

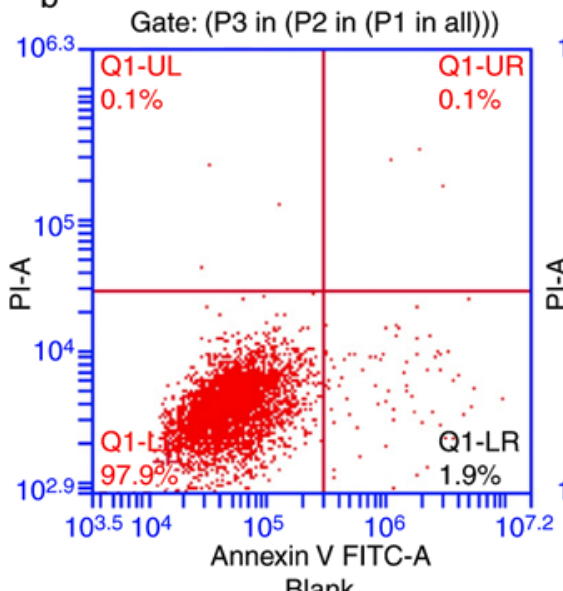

Blank

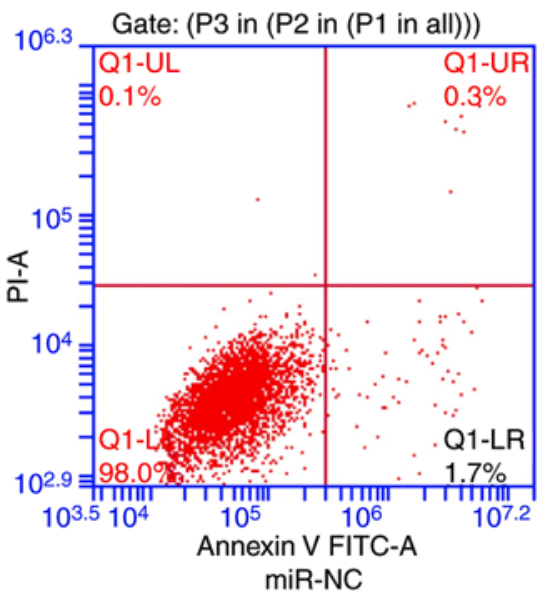

miR-NC

EC109

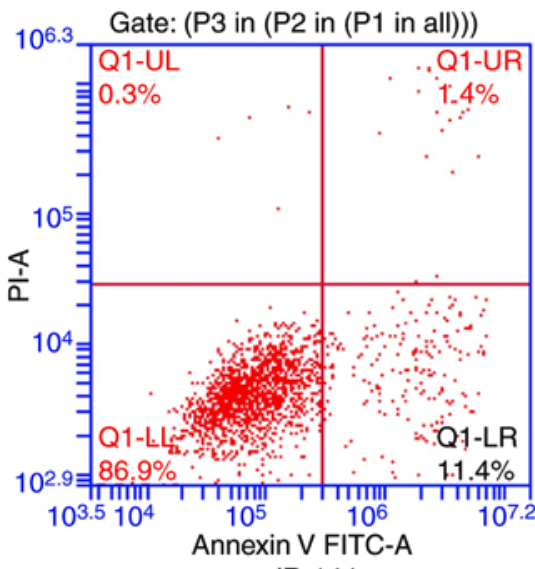

miR-144
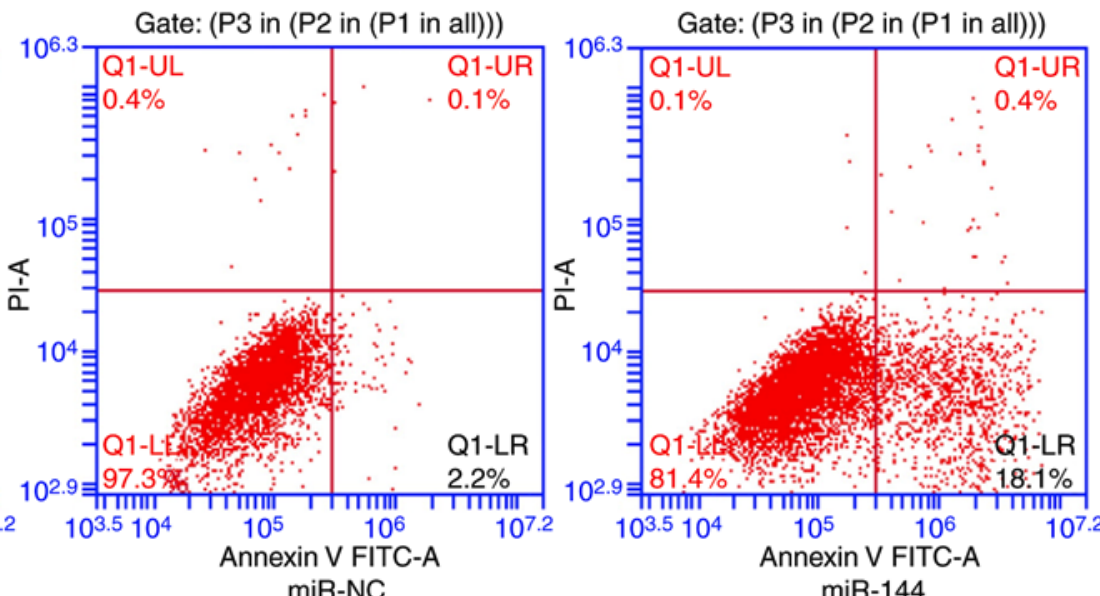

miR-144
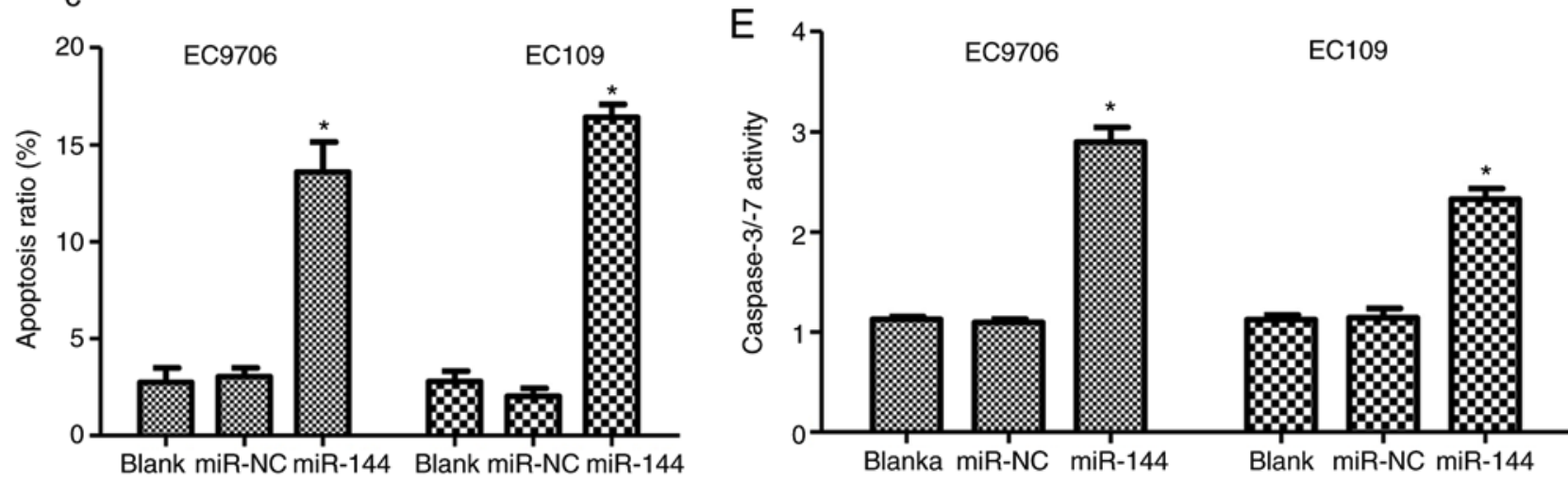

EC9706

EC109

Figure 2. Continued. miR-144 suppresses cell proliferation and tumor formation and promotes cell apoptosis in EC9706 and EC109 cells. (D) (a-c) Flow cytometric analysis assay revealed that miR-144 promotes cell apoptosis in ESCC cells. (E) Caspase-3/-7activity of miR-144 lentivirus transfected cells was almost 2 -fold as much as the miR-NC group and the blank group. "P $<0.05$ vs. control. miR, microRNA; ESCC, esophageal squamous cell carcinoma; $\mathrm{NC}$, negative control.

miR-144 promotes cell apoptosis in ESCC cells. The results of the flow cytometry analysis revealed that the proportion of apoptotic cells (Q1UR + Q1LR) in the miR-144 group was significantly higher than that of the miR-NC group and the blank group for both EC109 and EC9706 cells, which revealed that miR-144 overexpression effectively increased the number of apoptotic cells (Fig. 2D-a and c). Caspase-3/-7 activity apoptosis assay also demonstrated that the caspase-3/-7 activity of miR-144 lentivirus transfected cells was almost 2-fold as much as the miR-NC group and the blank group (Fig. 2E).
miR-144 inhibits tumor growth in vivo. In order to further investigate the role of miR-144 on ESCC, an in vitro investigation was performed. EC9706 cells transfected with miR-144 overexpression lentivirus and the control cells were injected into nude mice. Compared with the control groups, miR-144 overexpression markedly inhibited tumor volume, which indicated that miR-144 could inhibit ESCC tumor growth in vivo (Fig. $3 \mathrm{~A}$ and $\mathrm{B}$ ). The largest tumor diameters observed were $1.1,1.0$ and $0.5 \mathrm{~cm}$ in the blank group, miR-NC group and miR-144 group, respectively. Furthermore, the results of 

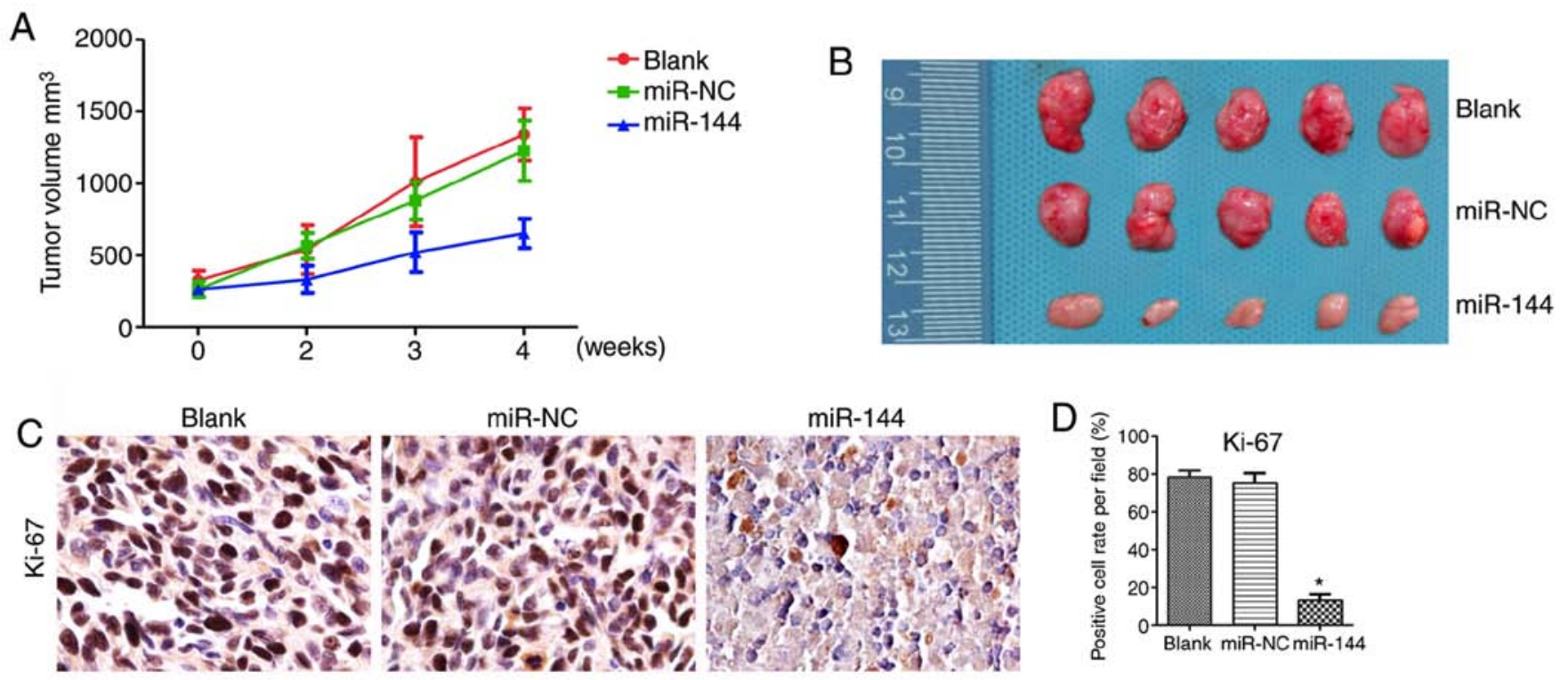

Figure 3. miR-144 inhibits tumor growth in vivo. (A) Overexpression of miR-144 significantly inhibited tumor volume compared with the control groups. (B) Images of the tumors from each group. (C) IHC staining data of Ki-67 on in vivo samples. (D) Quantification of IHC staining. ${ }^{*} \mathrm{P}<0.05$ vs. control. miR, microRNA; IHC, immunohistochemistry; NC, negative control.

IHC staining revealed that miR-144 inhibited Ki-67 expression in vivo (Fig. 3C and D).

miR-144 inhibits ESCC by targeting TIGAR. In order to verify the target gene of miR-144 in human ESCC, the following experiments were performed. First, the predicted binding sites of miR-144 in the 3'-UTR of TIGAR mRNA were revealed according to the results from the Targetscan analysis. Dual luciferase reporter gene assays revealed that miR-144 effectively weakened the luciferase activity of TIGAR wild-type by $\sim 50 \%$ ( $\mathrm{P}<0.05)$. However, miR-144 did not affect the luciferase activity of TIGAR with mutant type 3'UTR (Fig. 4A). PCR analysis confirmed the successful knockdown of TIGAR using the si-TIGAR (Fig. S1). Although the miR-144 group was demonstrated to exhibit a significant inhibiting effect on EC9706 cell proliferation, CCK-8 analysis revealed that the proliferation of EC9706 cells was also significantly inhibited when TIGAR was inhibited by si-TIGAR, when compared with the blank control ( $\mathrm{P}<0.05$; Fig. 4B). Similar results were also observed when using the tumor colony formation assay and the flow cytometry analysis (Fig. 4C-E). Collectively, these results indicated that TIGAR was a direct target of miR-144.

\section{Discussion}

Despite advances having been made regarding the treatment of ESCC, the molecular mechanism underlying the development of ESCC remains largely unknown, and patient overall survival time in the disease remains far from satisfactory $(2,6,29,30)$, which demonstrates an urgent need to identify novel molecular markers associated with ESCC and to facilitate the development of an effective diagnostic and therapeutic strategy for ESCC.

In the present study, miR-144 expression was observed to be downregulated in human ESCC tissues. Low miR-144 expression levels in tumors were significantly associated with metastasis and TNM staging in patients with ESCC, which revealed a possible association between miR-144 and ESCC tumorigenesis. The results of the present study demonstrated that the overexpression of miR-144 suppressed cell proliferation, and enhanced apoptosis in ESCC lines EC9706 and EC109. In addition, overexpression of miR-144 effectively inhibited tumor cell growth in vivo. Furthermore, it was revealed that miR-144 directly targeted TIGAR in ESCC cells. By decreasing the level of TIGAR using si-TIGAR, similar outcomes were observed to those with the overexpression of miR-144. These results indicated that miR-144 inhibits cell proliferation and invasion by directly targeting TIGAR in ESCC.

Various miRNAs have been reported to be aberrantly expressed in a number of different types of human cancer, including miR-144 (3,29,31). miR-144 has been widely researched and reported to be an important regulator of tumor development. However, the roles of miR-144 in human cancer are currently controversial (32-38), despite numerous reports having demonstrated that miR-144 is downregulated in a number of different types of human cancer, and that it may act as a tumor suppressor (19-22). There are studies demonstrating that miR-144 can promote cell proliferation of HeLa cells (39) and nasopharyngeal carcinoma cells (40). A recent study by Gao et al (21) demonstrated that the miR-144/451 cluster may serve an important role in the progression of ESCC and may be considered as a biomarker for the detection of ESCC at an early stage of disease. In their study, miR-144 was reported to be downregulated in ESCC tissues and cell lines, and overexpression of miR-144 was revealed to inhibit the proliferation of ESCC cells in vitro (21). In the present study, the gene expression profiles of miR-144 in human ESCC tissues were investigated using RT-qPCR. It was revealed that miR-144 was significantly downregulated in ESCC tissues when compared with the adjacent normal non-tumor tissues, which 
A

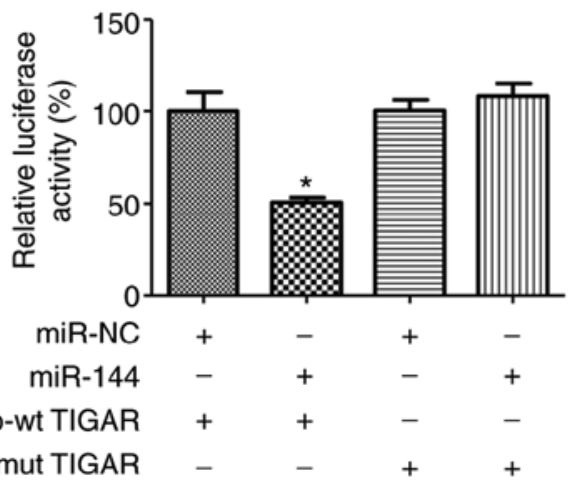

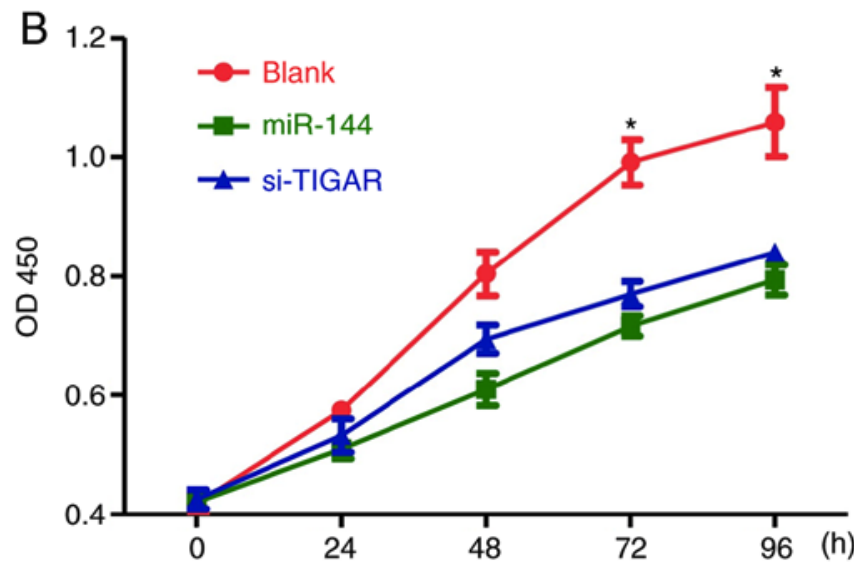
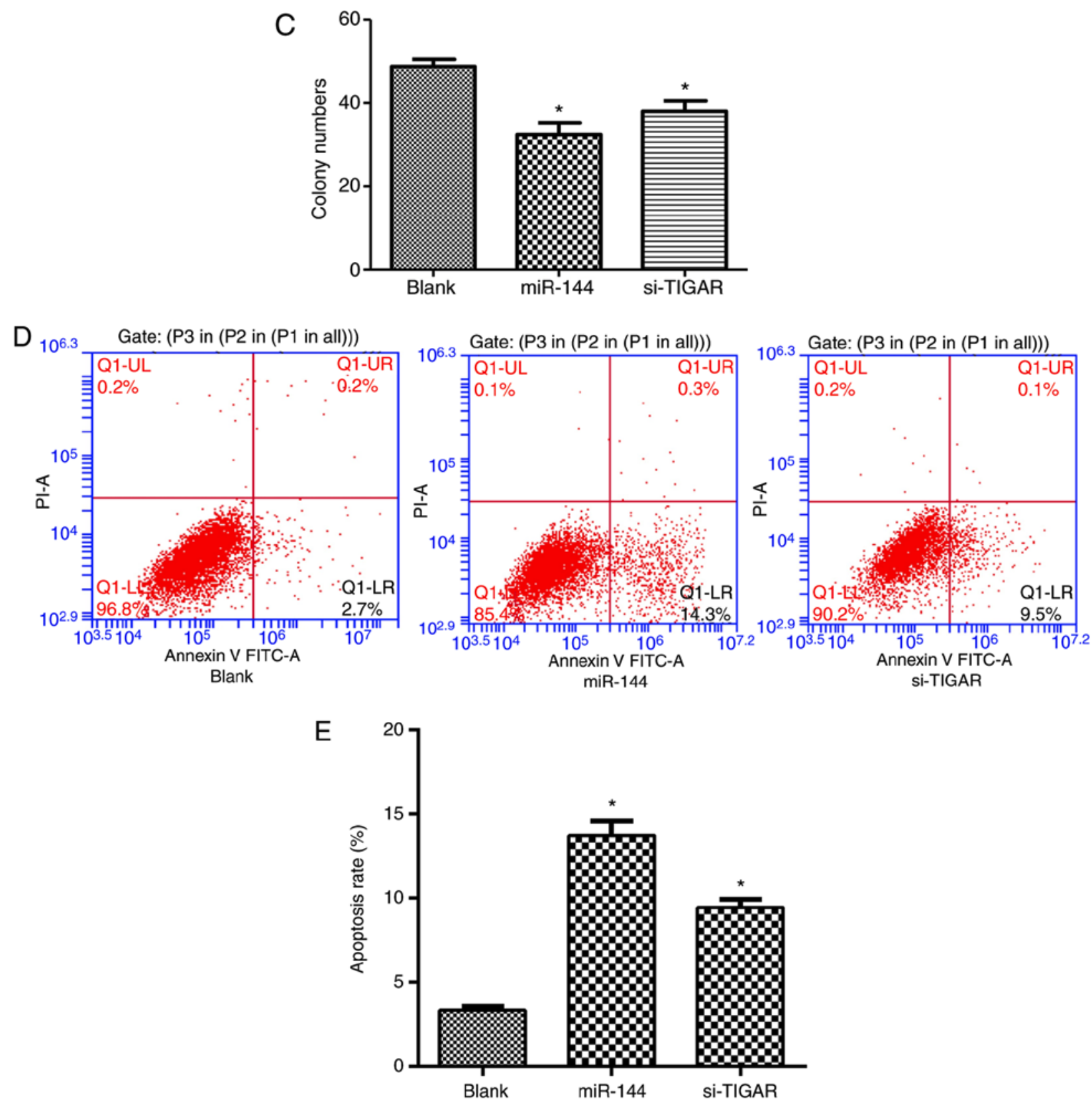

Figure 4. miR-144 inhibits ESCC by targeting TIGAR. (A) Dual luciferase reporter gene assays revealed that miR-144 effectively weakened the luciferase activity of TIGAR wild-type by $\sim 50 \%$, while miR-144 did not affect the luciferase activity of TIGAR with mutant type 3'UTR. (B) Cell Counting Kit-8 analysis revealed that the proliferation of EC9706 cells was significantly inhibited when TIGAR was inhibited by si-TIGAR, when compared with the blank control. (C) Tumor colony formation assay revealed that the proliferation of EC9706 cells was significantly inhibited when TIGAR was inhibited by si-TIGAR, when compared with the blank control. (D) Flow cytometric analysis revealed that the apoptosis rate of EC9706 cells was significantly increased when TIGAR was inhibited by si-TIGAR, when compared with the blank control. (E) Quantification of the flow cytometric analysis assay. "P<0.05 vs. blank group. miR, microRNA; TIGAR, TP53-inducible glycolysis and apoptosis regulator; 3'UTR, 3'untranslated region; si, small interfering; NC, negative control. 
was consistent with the results of previous studies $(21,41)$. Furthermore, the in vitro results of miR-144 expression levels were confirmed by an in vivo evaluation.

It was also revealed in the present study that TIGAR was the direct downstream target gene of miR-144 in ESCC. Furthermore, it was demonstrated that siRNA-mediated TIGAR knockdown was able to obtain similar inhibitory outcomes as with the overexpression of miR-144. TIGAR is an inhibitor of glycolysis that is highly expressed in a number of different types of human carcinoma, and it is currently the only known phosphatase glycolytic modulator regulated by TP53 (42). Previous studies have demonstrated that TIGAR is an important factor in moderating stress levels, repairing DNA damage and inhibiting glycolysis. Silencing of TIGAR in glioblastoma cell lines was revealed to cause higher levels of Fru-2,6-P2 in these cells, increase glycolysis and lead to the accumulation of reactive oxygen species, which finally promoted cell death (43). In addition, the present study demonstrated that TIGAR expression was significantly upregulated in ESCC tissues and strongly connected with ESCC tumor size and TNM stage, which further confirmed the conclusion that the effects of miR-144 on ESCC are primarily based on the regulation of the TIGAR signaling pathway.

There were several limitations to the present study. First, the independent role of TIGAR in the development of ESCC was not investigated, and the expression levels of TIGAR in the animal model require confirmation in an in vivo model. Secondly, besides TIGAR, other miR-144 targets that have already been demonstrated to result in similar phenotypes (e.g., RUNX1, CEP55, etc.) should also be investigated in future studies, which may aid in the identification of, or a combination of, novel pathways responsible for the demonstrated phenotypes. In addition, although the association between TNM staging and miR-144, TIGAR expression were investigated in the present study, other parameters, such as lymph node metastasis, $T$ stage and vascular invasion were not investigated. Additional groups, such as miR-144+ overexpression of TIGAR were also not investigated, which would be useful in confirming the results of the present study and should be the focus of future studies. Finally, due to the follow-up time, there was limited ability to analyze the association between miR-144, TIGAR and patient survival time. Thus, further studies are required to elucidate these queries.

Overall, the present study revealed that miR-144 inhibits cell proliferation and invasion by directly targeting TIGAR in esophageal carcinoma.

\section{Acknowledgements}

Not applicable.

\section{Funding}

No funding was received.

\section{Availability of data and materials}

The datasets used and/or analyzed during the current study are available from the corresponding author upon reasonable request.

\section{Authors' contributions}

BZ designed the study. YM acquired the data. QW and LT analyzed and interpreted the data. LL performed the statistical analyses. YM wrote the manuscript. BZ reviewed the manuscript for intellectual content.

\section{Ethics approval and consent to participate}

The present study was approved by the Medical Ethics Committee of the Affiliated Hospital of Taishan Medical University. Written informed consent was provided by all patients that participated in the present study.

\section{Patient consent for publication}

Not applicable.

\section{Competing interests}

The authors declare that they have no competing interests.

\section{References}

1. Bai Y, Lin H, Fang Z, Luo Q, Fang Y, Su Y, Hu Q, Duan H, Chen F and Zhang ZY: Plasma microRNA-19a as a potential biomarker for esophageal squamous cell carcinoma diagnosis and prognosis. Biomark Med 11: 431-441, 2017

2. Cao Z, Zheng X, Cao L and Liang N: MicroRNA-539 inhibits the epithelial-mesenchymal transition of esophageal cancer cells by twist-related protein 1-mediated modulation of melanoma associated antigen A4. Oncol Res 26: 529-536, 2018

3. Cui XB, Peng H, Li RR, Mu JQ, Yang L, Li N, Liu CX, $\mathrm{Hu}$ JM, Li SG, Wei Y, et al: MicroRNA-34a functions as a tumor suppressor by directly targeting oncogenic PLCE1 in Kazakh esophageal squamous cell carcinoma. Oncotarget 8: 92454-92469, 2017.

4. Fong LY, Taccioli C, Jing R, Smalley KJ, Alder H, Jiang Y, Fadda P, Farber JL and Croce CM: MicroRNA dysregulation and esophageal cancer development depend on the extent of zinc dietary deficiency. Oncotarget 7: 10723-10738, 2016.

5. Chen M, Xia Y, Tan Y, Jiang G, Jin H and Chen Y: Downregulation of microRNA-370 in esophageal squamous-cell carcinoma is associated with cancer progression and promotes cancer cell proliferation via upregulating PIN1. Gene 661: 68-77, 2018.

6. Chen Z, Zhao L, Zhao F, Yang G and Wang J: MicroRNA-26b regulates cancer proliferation migration and cell cycle transition by suppressing TRAF5 in esophageal squamous cell carcinoma. Am J Transl Res 8: 1957-1970, 2016.

7. Dong W, Li B, Wang J, Song Y, Zhang Z, Fu C and Zhang P: Diagnostic and predictive significance of serum microRNA-7 in esophageal squamous cell carcinoma. Oncol Rep 35: 1449-1456, 2016.

8. Gao X, Wang X, Cai K, Wang W, Ju Q, Yang X, Wang H and Wu H: MicroRNA-127 is a tumor suppressor in human esophageal squamous cell carcinoma through the regulation of oncogene FMNL3. Eur J Pharmacol 791: 603-610, 2016.

9. He W, Feng J, Zhang Y, Wang Y, Zang W and Zhao G: microRNA-186 inhibits cell proliferation and induces apoptosis in human esophageal squamous cell carcinoma by targeting SKP2. Lab Invest 96: 317-324, 2016.

10. Ji HH, Hong L, Huang GL, Yin HX, Xu P, Luo SY and Song JK: Association between microRNA-196a2 rs11614913, microRNA-146a rs2910164, and microRNA-423 rs6505162 polymorphisms and esophageal cancer risk: A meta-analysis. Meta Gene 3: 14-25, 2015.

11. Wu N, Song Y, Pang L and Chen Z: CRCT1 regulated by microRNA-520 $\mathrm{g}$ inhibits proliferation and induces apoptosis in esophageal squamous cell cancer. Tumour Biol 37: 8271-8279, 2016. 
12. Yu Q, Liu Y, Wen C, Zhao Y, Jin S, Hu Y, Wang F, Chen L, Zhang B, Wang W, et al: MicroRNA-1 inhibits tumorigenicity of esophageal squamous cell carcinoma and enhances sensitivity to gefitinib. Oncol Lett 15: 963-971, 2018.

13. Bulibu J, Wang W, Tang Y, Li N and Saifuding K: Association between polymorphisms in the promoter region of microRNA-34b/c and the chemoradiotherapy efficacy for locally advanced esophageal squamous cell carcinoma in Chinese Han population. Pathol Oncol Res 25: 421-427, 2019.

14. Harada K, Baba Y, Ishimoto T, Kosumi K, Tokunaga R, Izumi D, Ohuchi M, Nakamura K, Kiyozumi Y, Kurashige J, et al: Suppressor microRNA-145 is epigenetically regulated by promoter hypermethylation in esophageal squamous cell carcinoma. Anticancer Res 35: 4617-4624, 2015.

15. Chan CM, Lai KK, Ng EK, Kiang MN, Kwok TW, Wang HK, Chan KW, Law TT, Tong DK, Chan KT, et al: Serum microRNA-193b as a promising biomarker for prediction of chemoradiation sensitivity in esophageal squamous cell carcinoma patients. Oncol Lett 15: 3273-3280, 2018

16. Dong S, Yin H, Dong C, Sun K, Lv P, Meng W, Ming L and He F: Predictive value of plasma MicroRNA-216a/b in the diagnosis of esophageal squamous cell carcinoma. Dis Markers 2016 1857067,2016

17. Fan YX, Bian XH, Qian PD, Chen ZZ, Wen J, Luo YH, Yan PW and Zhang Q: MicroRNA-125b inhibits cell proliferation and induces cell apoptosis in esophageal squamous cell carcinoma by targeting BMF. Oncol Rep 40: 61-72, 2018.

18. Berry MF: Esophageal cancer: Staging system and guidelines for staging and treatment. J Thorac Dis 6 (Suppl 3): S289-S297, 2014.

19. Liu L, Wang S, Chen R, Wu Y, Zhang B, Huang S, Zhang J, Xiao F, Wang $M$ and Liang Y: Myc induced miR-144/451 contributes to the acquired imatinib resistance in chronic myelogenous leukemia cell K562. Biochem Biophys Res Commun 425: 368-373, 2012.

20. Whitman SP, Maharry K, Radmacher MD, Becker H, Mrózek K, Margeson D, Holland KB, Wu YZ, Schwind S, Metzeler KH, et al: FLT3 internal tandem duplication associates with adverse outcome and gene- and microRNA-expression signatures in patients 60 years of age or older with primary cytogenetically normal acute myeloid leukemia: A Cancer and Leukemia Group B study. Blood 116: 3622-3626, 2010.

21. Gao Z, Liu R, Liao J, Yang M, Pan E, Yin L and Pu Y: Possible tumor suppressive role of the miR-144/451 cluster in esophageal carcinoma as determined by principal component regression analysis. Mol Med Rep 14: 3805-3813, 2016.

22. Wu W, Hou W, Wu Z, Wang Y, Yi Y and Lin W: miRNA-144 in the saliva is a genetic marker for early diagnosis of esophageal cancer. Nan Fang Yi Ke Da Xue Xue Bao 33: 1783-1786, 2013 (In Chinese).

23. Livak KJ and Schmittgen TD: Analysis of relative gene expression data using real-time quantitative PCR and the 2(-Delta Delta $\mathrm{C}(\mathrm{T})$ ) method. Methods 25: 402-408, 2001.

24. Agarwal V, Bell GW, Nam JW and Bartel DP: Predicting effective microRNA target sites in mammalian mRNAs. Elife 4, 2015

25. Lewis BP, Burge CB and Bartel DP: Conserved seed pairing, often flanked by adenosines, indicates that thousands of human genes are microRNA targets. Cell 120: 15-20, 2005.

26. Vlachos IS, Zagganas K, Paraskevopoulou MD, Georgakilas G, Karagkouni D, Vergoulis T, Dalamagas T and Hatzigeorgiou AG DIANA-miRPath v3.0: Deciphering microRNA function with experimental support. Nucleic Acids Res 43: W460-W466, 2015

27. Hu C, Lv L, Peng J, Liu D, Wang X, Zhou Y and Huo J: MicroRNA-375 suppresses esophageal cancer cell growth and invasion by repressing metadherin expression. Oncol Lett 13 . 4769-4775, 2017.

28. Zhang ZQ, Cao Z, Liu C, Li R, Wang WD and Wang XY: MiRNA-embedded ShRNAs for radiation-inducible LGMN knockdown and the antitumor effects on breast cancer. PLoS One 11: e0163446, 2016.
29. Dong S, Yin H, Dong C, Sun K, Lv P, Meng W, Ming L and He F: Corrigendum to 'Predictive value of plasma MicroRNA-216a/b in the diagnosis of esophageal squamous cell carcinoma'. Dis Markers 2017: 3437679, 2017.

30. Fong LY, Farber JL and Croce CM: Zinc intake, microRNA dysregulation, and esophageal cancer. Aging (Albany NY) 8: 1161-1162, 2016.

31. Cui H, Zhang S, Zhou H and Guo L: Direct downregulation of B-cell translocation gene 3 by microRNA-93 is required for desensitizing esophageal cancer to radiotherapy. Dig Dis Sci 62 : 1995-2003, 2017.

32. Fan JY, Yang Y, Xie JY, Lu YL, Shi K and Huang YQ: MicroRNA-144 mediates metabolic shift in ovarian cancer cells by directly targeting Glut1. Tumour Biol 37: 6855-6860, 2016.

33. Huang J, Shi Y, Li H, Yang M and Liu G: MicroRNA-144 acts as a tumor suppressor by targeting Rho-associated coiled-coil containing protein kinase 1 in osteosarcoma cells. Mol Med Rep 12: 4554-4559, 2015.

34. Huo F, Zhang C, He H and Wang Y: MicroRNA-144-3p inhibits proliferation and induces apoptosis of human salivary adenoid carcinoma cells via targeting of mTOR. Biotechnol Lett 38 409-416, 2016.

35. Jin J, Wang $Y, X u$ Y, Zhou X, Liu Y, Li X and Wang J: MicroRNA-144 regulates cancer cell proliferation and cell-cycle transition in acute lymphoblastic leukemia through the interaction of FMN2. J Gene Med 19, 2017.

36. Li B, Zhang S, Shen H and Li C: MicroRNA-144-3p suppresses gastric cancer progression by inhibiting epithelial-to-mesenchymal transition through targeting PBX3. Biochem Biophys Res Commun 484: 241-247, 2017.

37. Li D, Li H, Yang Y and Kang L: Long noncoding RNA urothelial carcinoma associated 1 promotes the proliferation and metastasis of human lung tumor cells by regulating MicroRNA-144. Oncol Res 26: 537-546, 2018.

38. Lin L, Zheng Y, Tu Y, Wang Z, Liu H, Lu X, Xu L and Yuan J: MicroRNA-144 suppresses tumorigenesis and tumor progression of astrocytoma by targeting EZH2. Hum Pathol 46: 971-980, 2015.

39. Cheng AM, Byrom MW, Shelton J and Ford LP: Antisense inhibition of human miRNAs and indications for an involvement of miRNA in cell growth and apoptosis. Nucleic Acids Res 33: 1290-1297, 2005

40. Zhang LY, Ho-Fun Lee V, Wong AM, Kwong DL, Zhu YH, Dong SS, Kong KL, Chen J, Tsao SW, Guan XY and Fu L: MicroRNA-144 promotes cell proliferation, migration and invasion in nasopharyngeal carcinoma through repression of PTEN. Carcinogenesis 34: 454-463, 2013.

41. Shao Y, Li P, Zhu ST, Yue JP, Ji XJ, Ma D, Wang L, Wang YJ, Zong Y, Wu YD and Zhang ST: MiR-26a and miR-144 inhibit proliferation and metastasis of esophageal squamous cell cancer by inhibiting cyclooxygenase-2. Oncotarget 7: 15173-15186, 2016.

42. Ko YH, Domingo-Vidal M, Roche M,Lin Z, Whitaker-Menezes D, Seifert E, Capparelli C, Tuluc M, Birbe RC, Tassone P, et al: TP53-inducible glycolysis and apoptosis regulator (TIGAR) metabolically reprograms carcinoma and stromal cells in breast cancer. J Biol Chem 291: 26291-26303, 2016.

43. Xu X, Liu C and Bao J: Hypoxia-induced hsa-miR-101 promotes glycolysis by targeting TIGAR mRNA in clear cell renal cell carcinoma. Mol Med Rep 15: 1373-1378, 2017.

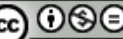

This work is licensed under a Creative Commons Attribution-NonCommercial-NoDerivatives 4.0 International (CC BY-NC-ND 4.0) License. 